\title{
1 Freshwater sponge hosts and their green algae 2 symbionts: a tractable model to understand intracellular 3 symbiosis
}

5 Chelsea Hall2,3, Sara Camilli3,4, Henry Dwaah2, Benjamin Kornegay2, Christine A. Lacy2,

6 Malcolm S. Hill1,2§, April L. Hill1,2§

8 1Department of Biology, Bates College, Lewiston ME, USA

9 2Department of Biology, University of Richmond, Richmond VA, USA

10 3University of Virginia, Charlottesville, VA, USA

11 4Princeton University, Princeton, NJ, USA

13 §Present address: Department of Biology, Bates College, Lewiston ME USA

14 Corresponding author:

15 April L. Hill

1644 Campus Ave, Lewiston, ME 04240, USA

17 Email address: ahill5@bates.edu

18

19

20

21 


\section{Abstract}

28 In many freshwater habitats, green algae form intracellular symbioses with a variety of

29 heterotrophic host taxa including several species of freshwater sponge. These sponges perform

30 important ecological roles in their habitats, and the poriferan:green algae partnerships offers

31 unique opportunities to study the evolutionary origins and ecological persistence of

32 endosymbioses. We examined the association between Ephydatia muelleri and its chlorophyte

33 partner to identify features of host cellular and genetic responses to the presence of intracellular

34 algal partners. Chlorella-like green algal symbionts were isolated from field-collected adult $E$.

35 muelleri tissue harboring algae. The sponge-derived algae were successfully cultured and

36 subsequently used to reinfect aposymbiotic E. muelleri tissue. We used confocal microscopy to

37 follow the fate of the sponge-derived algae after inoculating algae-free E. muelleri grown from

38 gemmules to show temporal patterns of symbiont location within host tissue. We also infected

39 aposymbiotic E. muelleri with sponge-derived algae, and performed RNASeq to study

40 differential expression patterns in the host relative to symbiotic states. We compare and contrast

41 our findings with work in other systems (e.g., endosymbiotic Hydra) to explore possible

42 conserved evolutionary pathways that may lead to stable mutualistic endosymbioses. Our work

43 demonstrates that freshwater sponges offer many tractable qualities to study features of

44 intracellular occupancy and thus meet criteria desired for a model system. 
Introduction

47 A watershed moment for life on this planet involved the successful invasion of, and persistent residence within, host cells by bacterial symbionts (i.e., proto-mitochondria and protochloroplasts), which opened evolutionary pathways for multicellular organisms (Margulis, 1993). Indeed, endosymbioses that involve benefits for both interacting partners are abundant in modern

51 ecosystems (Douglas, 2010; Bordenstein \& Theis, 2015). Intracellular symbioses involving

52 phototrophic symbionts and heterotrophic hosts are particularly important given that they support

53 many ecological communities. For example, Symbiodinium populations harbored by cnidarian

54 and other invertebrate hosts energetically subsidize the entire coral reef ecosystem (Stambler,

55 2011). In many freshwater habitats, green algae (e.g., Chlorella spp.) form intracellular

56 symbioses with a variety of heterotrophic host taxa, and these types of "nutritional mutualisms"

57 (Clark et al., 2017) are essential in aquatic habitats (Smith \& Douglas, 1987; Reiser, 1992).

Despite their importance, many facets of the molecular and cellular interactions that allow longterm partnerships remain obscure for a range of phototroph:heterotroph symbioses (Hill \& Hill 2012). To date, our understanding of freshwater:algal intracellular symbiosis has largely been informed by two Chlorella-based symbioses found in Paramecium and Hydra host backgrounds

63 (e.g., Kodama \& Fuhishima, 2010; Kovacevic, 2012). Hydra:Chlorella symbioses were among

64 the first animal systems to conclusively demonstrate the transfer of photosynthetically-fixed

65 carbon from the symbiont to the host (Muscatine \& Hand, 1958) and Paramecium:Chlorella

66 symbioses have long been known to benefit host growth (Karakasian, 1963). While Chlorella-

67 based symbioses have been the most studied in both Paramecium and Hydra, it is clear that

68 photosynthetic green algal symbionts other than Chlorella are also predominant in many species 
69 and that intracellular algal symbioses have evolved multiple times over the course of evolution

70 (Hoshina \& Imamura, 2008, Rajevic et al., 2015). Molecular and cellular tools have shed further

71 light on the symbioses revealing that a highly coordinated series of cellular and molecular events

72 transpires as Chlorella are taken up by Paramecium (Kodama \& Fujishima, 2010), and a unique

73 set of genes are up and down regulated in the host in response to establishment of the symbiosis

74 in Paramecium with and without Chlorella symbionts (Kodama et al., 2014). Among the

75 mechanisms that appear to be regulated during endosymbiosis, glutamate and glutamine

76 biosynthesis has been speculated to play roles in nitrogen metabolism. For example, He et al.,

77 (2019) demonstrated that Paramecium bursaria regulate abundance of their symbionts through

78 glutamine supply. Distinct gene expression patterns during endosymbiotic interactions between

79 two species of Hydra and their algal symbionts has also been revealed (Ishikawa et al., 2016) and

80 interestingly, glutamine synthesis seems to play a key role in this symbiosis as well (Hamada et

81 al., 2018).

83 Obligacy that involves tight integration between partners is the pinnacle of coevolutionary

84 specialization (e.g., Amann et al., 1997). However, the initial interactions involving intracellular

85 occupancy likely involved some degree of ephemerality (Strehlow et al. 2016), and even for

86 well-studied symbioses, specific factors that permit long-term residency of a symbiont within a

87 host cell often remain obscure (Clark et al., 2017). A comparative approach is especially useful if

88 we hope to understand the forces that shape obligate and long-lasting mutualistic symbioses.

89 Both of the classic examples of endosymbiosis mentioned previously (Hydra and Paramecium)

90 offer opportunities for comparative analysis of the cellular events that permit establishment and

91 stability of intracellular residency (Hill, 2014). Bosch, Guillemin \& McFall-Ngai (2019) recently 
92 highlighted the development and use of several laboratory symbiosis model systems that will

93 help construct a more complete picture of host-microbe interactions including several early

94 branching animals (e.g., Nematostella vectensis, Aiptasia pallida, Hydra vulgaris). They argue

95 that interrogating a variety of "evolutionary 'experiments' in symbiosis" will shed light on the

96 mechanisms and diversity of these interactions and lead to better understanding of how animals

97 have evolved, making the case that future studies should include identifying mechanisms for

98 symbiosis in sponge holobionts.

99

100 Freshwater sponges from several genera harbor green algal species and these partnerships were

101 objects of study by early scientists interested in symbiosis (Brøndsted \& Brøndsted, 1953;

102 Brøndsted \& Løvtrup, 1953; Muscatine, Karakashian \& Karakashian, 1967; Gilbert \& Allen,

103 1973a; Gilbert \& Allen, 1973b; Wilkinson, 1980). The focus of much of the work has been on

104 sponge:Chlorella partnerships and has centered on the ecological importance of photosynthetic

105 sponges in freshwater ecosystems (e.g., Williamson, 1977; Williamson, 1979; Frost \&

106 Williamson, 1980), but effort has waned over the last 25 years. Several features of freshwater

107 sponge biology make them excellent systems for studying the molecular and cellular pathways

108 required for successful symbiosis yet freshwater sponge symbioses are poorly represented in the

109 modern algal-based symbiosis literature. The emergence of molecular tools offers renewed

110 opportunities to study sponge-based symbiotic systems, and freshwater sponges offer many

111 tractable qualities of a model system to study intracellular symbioses (Kenny et al., 2019; Kenny

112 et al., 2020). 
114 We demonstrate here that the sponge E. muelleri is an excellent model to study symbiosis. The

115 genus Ephydatia belongs to the Spongillidae, a species rich family of exclusively freshwater

116 haplosclerid demosponges. It has a pancontinental distribution, which may be due at least in part

117 to transportation in guts (McAuley and Longcore 1988) or on feathers (Manconi \& Pronzato,

118 2016) of foraging waterfowl. It produces diapausing cysts (i.e., gemmules) that can withstand

119 freezing and be stored at $-80 \mathrm{oC}$ (Leys, Grombacher \& Hill, 2019), and thousands of clonal

120 individuals can be cultured at room temperature with minimal lab equipment (Barbeau, Reiswig

$121 \&$ Rath, 1989). Due to the facultative nature of the sponge:symbiont partnerships, the green algal

122 symbiont can often be easily cultured outside of the host, and, as we show here, sponges can

123 grow with and without the symbionts. Recently, a high quality E. muelleri genome was

124 sequenced with chromosomal-level assembly and RNASeq data for four developmental stages

125 (Kenny et al., 2020). E. muelleri is also amenable to a variety of cellular, genetic, and molecular

126 approaches that allow researchers to study gene function (e.g., Windsor \& Leys, 2010; Rivera et

127 al., 2011: Schenkelaars et al., 2016; Schippers \& Nichols 2018; Windsor et al., 2018; Hall et al.,

128 2019). These aspects of sponge:algal cultivation along with the molecular resources make $E$.

129 muelleri a tractable model system to study host:symbiont integration and specialization at a

130 cellular and genetic level to identify mechanisms that shape integration between hosts and

131 symbionts.

133 Here we evaluate host:symbiont interactions by examining the fate of sponge-derived Chlorella-

134 like green algae introduced to aposymbioitc sponges recently hatched from gemmules. We

135 identify putative genetic pathways involved with establishing the endosymbiosis through 
136 RNASeq analysis and we discuss the implications of this work in light of growing interest in

137 understanding general mechanisms that may guide symbiotic interactions.

\section{Materials and Methods}

140 Sponge and Algal collection

141 Sponge gemmules were collected in the winter months from shallow, rocky streams at the base

142 of dams in Richmond, VA in Bryan Park (37.598047, -77.468428) under Virginia Department of

143 Game and Inland Fisheries Permit \#047944. Gemmule-containing sponges were located on the

144 undersides of rocks, and samples were transported on ice in foil-wrapped, $50 \mathrm{ml}$ conical tubes. In

145 the lab, gemmule-containing sponge tissue was placed in cold 1X Strekal's solution (Strekal \&

146 McDiffett, 1974) in a petri dish, and under a microscope illuminated with low light, gemmules

147 were separated from residual adult skeletal material. Isolated gemmules were washed in a weak

148 hydrogen peroxide solution (2\%) before being stored at $4^{\circ} \mathrm{C}$ in $1 \mathrm{X}$ Strekal's or in $20 \% \mathrm{DMSO}$ at -

$14980^{\circ} \mathrm{C}$ (Leys, Grombacher \& Hill, 2019).

151 Algae-bearing sponges were identified in summer months based on their bright green coloration, 152 and sponges were returned to the lab for algal isolation. A small piece $(\approx 1 \mathrm{~cm} 3)$ of clean tissue

153 was removed from the sponge, and then washed multiple times in 1X Strekal's solution. Cleaned

154 sponge tissue was then ground in 1X Bold Basal Medium (BBM; Sigma-Aldrich, Milwaukee,

155 WI) in a clean, acid-washed mortar and pestle. Algae in the resultant slurry were allowed to

156 precipitate and the supernatant was removed and replaced with fresh 1X BBM. This process was

157 repeated multiple times to create an algal-enriched solution. Once nearly all visible sponge

158 material was removed, $1 \mu 1$ of the algal suspension was added to $200 \mathrm{ml}$ of sterile BBM. Algal 
159 growth was obvious within 1 week. Algal cultures were subsequently plated onto BBM agar

160 plates for the isolation of individual algal colonies. Algal lines were grown continuously in either

161 Basal Medium (Sigma-Aldrich, Milwaukee, WI) or in Modified Bolds 3N Medium (UTEX,

162 Austin, TX).

163

164 Algal cultures and Identification

165 Algae were propagated at $\pm 25 \mathrm{oC}$ under fluorescent light for 16 hour per day. DNA from cultured

166 algae was isolated using the CTAB procedure, and 18S rDNA was sequenced. PCR

167 amplification of 18s rDNA was done using protist specific molecular barcoding primers E528F,

168 N920R, GF, GR, BR, and ITS055R (Marin et al, 1998; Marin et al, 2003). PCR conditions

169 included 4 min at $94^{\circ} \mathrm{C} ; 30$ cycles of $30 \mathrm{sec}$ at $94^{\circ} \mathrm{C}, 30 \mathrm{sec}$ at $55^{\circ} \mathrm{C}$, and $45 \mathrm{sec}$ at $72^{\circ} \mathrm{C}$. A final

170 elongation step of 2 min at $72^{\circ} \mathrm{C}$ was included. PCR products were separated on a $1 \%$ agarose

171 gel to verify amplification. Amplicons were cleaned using the QIAquick PCR Purification Kit

172 (Qiagen, Hilden, Germany) and sequenced. Additional markers for identification of Chlorella

173 spp. isolates for nuclear SSU and chloroplast SSU were also used (Wu, Hseu \& Lin, 2001) and

174 products were sequenced as described. All sequences are provided in File S1.

176 Algal infection of sponges

177 Ephydatia muelleri was grown from gemmules in $1 \mathrm{X}$ Strekals in 6 well plates over a three to

178 five-day period, which corresponded to the development of a mature canal system with osculum

179 and evidence of active pumping (Leys, Grombacher \& Hill, 2019). Live sponge-derived algal

180 cells were introduced into the water surrounding the sponge. We initiated all infections with

181 130,000 algal cells ml-11X Strekal's harvested during the logarithmic portion of their growth 
182 phase. We estimated cell densities and population growth characteristics using optical density

183 (OD) measurements at $425 \mathrm{~nm}$ and $675 \mathrm{~nm}$, which had been correlated with actual cell counts

184 determined with a hemocytometer. Algae were slowly pipetted around and above the tissue to

185 inoculate sponges. Infected sponges were placed under a 12:12 light:dark exposure.

187 Microscopy

188 For confocal microscopy, sponges were grown in $35 \mathrm{~mm}$ glass bottom dishes (MatTek Life

189 Sciences) and sponge tissue with and without algae was fixed in 4\% paraformaldehyde and 1/4

190 Holtfreter's Solution overnight at 40 C. Tissue was washed three times in 1/4 Holtfreter's

191 Solution, permeabilized with $0.1 \%$ Triton X-100/PBS for three minutes, and washed three times

192 in PBS. Tissue was stained with Hoescht 33342 (1:200 dilution, Thermo Fisher Scientific,

193 Waltham, MA) and Phalloidin Alexa 488 (1:40 dilution, Thermo Fisher Scientific, Waltham,

194 MA) in PBS and incubated in the dark for 20 minutes, washed three times in PBS and imaged

195 imaged using an Olympus FV1200 laser scanning microscope using FluoView software.

197 For electron microscopy, sponge samples infected with algae were fixed in $2.5 \%$ glutaraldehyde

198 in sterile filtered water for 1 hour at room temperature and then overnight at $4^{\circ} \mathrm{C}$. Fixed samples

199 were washed in $0.2 \mathrm{M}$ cacodylate buffer $(\mathrm{pH} 7.4)$ and postfixed with $1 \%$ OsO4 and 1\% Uranyl

200 acetate. Samples were dehydrated in an ethanol series, infiltrated in propylene oxide, and

201 embedded in Embed 812 plastic resin. After polymerization, $1 \mathrm{~mm}$ sections were cut and treated

202 for 1 hour in 4\% hydrofluoric acid:76\% ethanol to dissolve spicules. These sections were then re-

203 dehydrated, re-infiltrated, and re-embedded following the protocol described above. Ultrathin 
204 sections were stained with uranyl acetate and quick lead. Micrographs were taken using a JEOL

2051010 transmission electron microscope.

207 RNA isolation, library construction, and sequencing

208 Sponges were grown from gemmules in 1X Strekal's to the stage where a functioning osculum

209 had developed. To triplicate samples of these sponges ( $20-30$ sponges per treatment), we added

210 live algal cells (130,000 Chlorella ml-1) or no algae as treatments. Tissue was collected after 24

211 hours of exposure to algae, washed several times to remove algae from the surrounding water

212 and surfaces, and either stored at $-80{ }^{\circ} \mathrm{C}$ after RNAlater treatment (Thermo Fisher Scientific,

213 Waltham, MA) or processed immediately for RNA. Total RNA was isolated using the animal

214 tissue RNA purification kit (Norgen Biotek, Thorold, Ontario, Canada). Total RNA was sent to

215 LC Sciences (Houston, TX) where RNA integrity was checked with Agilent Technologies 2100

216 Bioanalyzer (Agilent, CA). Ribosomal RNA was removed at LC Sciences using Ribo-Zero

217 ribosomal RNA reduction, followed by fragmentation with divalent cation buffers in elevated

218 temperature. Sequencing libraries were prepared by LC Sciences following Illumina's TruSeq-

219 stranded-total-RNA-sample preparation protocol (Illumina, San Diego, USA). Quality control

220 analysis and quantification of the sequencing library were performed using Agilent Technologies

2212100 Bioanalyzer High Sensitivity DNA Chip. Paired-ended sequencing was performed on

222 Illumina's NovaSeq 6000 sequencing system by LC Sciences.

224 Transcript assembly and analysis

225 Cutadapt 1.10 (Martin, 2011) and proprietary perl scripts (LC Sciences) were used to remove the

226 reads that contained adaptor contamination, low quality bases and undetermined bases. Sequence 
227 quality was verified using FastQC 0.10 .1

228 (http://www.bioinformatics.babraham.ac.uk/projects/fastqc/). Bowtie 2 (Langmead \& Salzberg, 229 2012) and HISAT 2.0 (Kim, Langmead \& Salzberg, 2015) were used to map reads to the 230 reference genome of E. muelleri (Kenny et al., 2020). The mapped reads (bam format) of each

231 sample were assembled using StringTie (Pertea et al., 2015). All transcriptomes from 6 samples

232 were merged to reconstruct a comprehensive transcriptome using perl scripts and gffcompare 233 (https://github.com/gpertea/gffcompare/). After the final transcriptome was generated, StringTie 234 (Pertea et al., 2015) and edgeR (Robinson, McCarthy \& Smyth, 2010) were used to estimate the 235 expression levels (FPKM) of all transcripts and genes across all replicate samples. mRNAs with $236 \log 2$ (fold change) $>1$ or $\log 2$ (fold change) $<-1$ and with statistical significance where the p237 value was $<0.05$ were considered to be differentially expressed at a significant level. Gene 238 Ontology (GO) and KEGG annotation and enrichment analysis

\section{Results}

241 Algal symbionts can be cultivated outside of freshwater sponge hosts

242 Freshwater sponges from the field are observed with and without symbionts, even within the 243 same individual, depending on growth locations and exposure to light (Fig. 1). Symbiotic algae

244 were isolated from Ephydatia muelleri, cultured, and DNA sequencing indicated that the isolate 245 belongs to the Chlorelleaceae (File S1). The strain is Chlorella-like in morphology, grows well in 246 commercially available algal media across a range of temperatures $\left(16 \mathrm{o}_{\mathrm{C}}\right.$ to $\left.25 \mathrm{o} \mathrm{C}\right)$ and light:dark 247 regimes $(12: 12,16: 8,24: 0)$. Due to its easily culturable nature, we have continuously grown this 248 strain for more than five years in the lab. Our Chlorella-like isolate reached a stationary phase of 249 growth (approximately $1.0 \times 108$ cells $/ \mathrm{ml}$ ) by 15 days when grown under the standard conditions 
used for growing freshwater sponges in the lab $\left(22-23_{\circ} \mathrm{C}, 16: 8\right.$ light:dark). The algae also grew well on BBM plates and individual colonies were used to make frozen stocks of the algal strain.

253 Sponge-derived algal symbionts stably infect aposymbiotic E. muelleri

254 Aposymbiotic E. muelleri sponges were hatched from gemmules and grown to full development

255 at stage 5 (Kenney et al., 2020). At this point, sponge-derived Chlorella-like symbionts in

256 exponential growth phase were added to the media. The infected sponges had extensive canal

257 systems and functioning oscula (Fig. 2). The majority of algal cells captured by E. muelleri

258 appeared to be located in intracellular compartments as observed by confocal microscopy (Fig.

259 3). Evidence of the establishment of intracellular residence by the algae was apparent within 4

260 hours of infection (Fig. 4A). At the 24 hour time point, we observed many sponge host cells that

261 harbored single or multiple algae within a single cell (Fig. 4B \& C; Fig. 5). Persistence of algae

262 within host cells through 6 days was obvious, though we observed that algae-containing sponge

263 cells shifted location and were concentrated around and adjacent to choanocyte chambers (Fig.

$2643 \mathrm{D})$.

RNA Sequencing, assembly, and genome mapping

267 Six cDNA libraries, three from aposymbiotic E. muelleri and three from E. muelleri 24 hours

268 post-infection with sponge-derived algae, were constructed and sequenced on the Illumina HiSeq

2694000 platform. Quality control and read statistic data for each sample are given in Table S1, with

270 results shown before and after read cleaning. Sequencing quality was exceptionally good, with

271 high (>98\%) Q30\% observed for all samples. The least well-recovered sample was EmInf3, with

$2727.54 \mathrm{Gbp}$ sequenced, and the most-sequenced sample, EmInf1, contained 10.32 Gbp. In all cases, 
273 a good level of sequencing depth was observed for three samples per stage. A total of $65,377,412$

274 raw reads with a Q20 value of $99.98 \%$ were generated for the aposymbiotic sponges and

$27559,214,624$ raw reads with a Q20 value of 99.98\% were generated for the symbiotic sponges.

276 After removing the low-quality sequences, short reads and ambiguous nucleotides, the remaining

277 valid reads were $63,552,928$ for the aposymbiotic treatment and 57,705,518 for symbiotic

278 treatments. For all replicate samples, good mapping results were observed to the reference

279 genome (Kenney, 2020). In any sample, no fewer than $56.50 \%$ of all reads could be mapped to

280 the E. muelleri genome and 36,771,764 (57.87\%) mapped reads and 22,562,710 (35.5\%) unique

281 reads were obtained for the aposymbiotic sequences while 33,145,990 (57.36\%) mapped reads

282 and 20,718,294 (35.96\%) unique reads were found for the symbiotic sequences (Table S2). We

283 do not have a genome sequence available for the sponge-derived Chlorella-like algae and thus

284 were not able to map algal transcripts from the RNASeq data set at this time. A future goal is to

285 obtain this genome sequence in order to begin to understand gene expression patterns from the

286 symbionts perspective. The number of raw reads mapped to each E. muelleri gene or transcript is

287 given in Table S3. Of the reads that map to the genome, greater than $70 \%$ of reads were placed in

288 exonic regions for all samples and less than $1 \%$ of the RNASeq reads mapped intergenically

289 (Fig. S1).

290

291 Normalizing of expression units was performed using FPKM for both gene and transcript

292 expression and FPKM interval chart and density graphs comparing overall gene expression

293 between samples (Fig. S2, Supp Table 4) reveal that variation in expression between samples is

294 low and distinct distributions are nearly the same for each sample. This indicates that the quality

295 of data obtained by sequencing was reliable for further analysis. Even though we do not yet have 
an available reference genome for the native sponge-derived algae or for the possible bacterial

297 symbionts present in our dataset, we believe that the overall transcriptome data sets will be of

298 interest to others who study bacterial symbionts or are interested in non-coding RNA as we used

299 total RNA sequencing to capture a broader range of gene expression changes (i.e., transcripts in

300 both coding and non-coding RNA). We also used RNA depletion rather than poly-A tail

301 selection.

302

Symbiosis-Related E. muelleri genes revealed by $R N A S e q$

304 To understand the genetic regulation of symbiont acquisition and maintenance from the host

305 perspective, we examined differential gene expression at 24 hours post-infection between

306 sponges grown without algal symbionts and those that were infected with sponge-derived

307 Chlorella-like symbionts. Analysis of gene expression profiles demonstrated 429 sponge genes

308 were significantly altered $(\log 2>1 ; \mathrm{p}<0.05)$ between aposymbiotic and symbiotic sponges, of

309 which 194 genes were upregulated during symbiont acquisition and 235 were downregulated

310 (Fig. 6, File S2, Fig. S3). Transcript expression profiles demonstrated a similar pattern (Fig. S4).

311 Among the genes with increased expression in symbiont infected sponges, 39\% were either

312 novel transcripts of unknown function or containing sequences or domains found in other

313 organisms, but otherwise uncharacterized proteins. The genes with increased expression in

314 aposymbiotic sponges that represent novel or uncharacterized proteins represented $46 \%$ of the

315 dataset.

317 Among the enriched Gene Ontology (GO) categories revealed by the analysis, we found

318 biological process categories to be enriched for those related to DNA catabolic processes and 
oxidation-reduction processes. Within the cellular component category, cytoplasm, nucleus, and membrane components were enriched. The molecular function categories included

321 deoxyribonuclease activity, ATP binding, and metal ion binding (Fig. S5). GO enrichment

322 analysis revealed several processes including monooxygenase activity and related

323 oxidoreductase activity. Chitin related activities, scavenger receptor activity, receptor mediated

324 endocytosis, DNA catabolic process, deoxyribonucleic acid activity, and multiple aspects of

325 copper ion binding, import, and export were also enriched (Fig. 7). Using KEGG, we identified a

326 variety of enriched pathways, including arachidonic acid, glutathione metabolism, and

327 metabolism of molecules by cytochrome $\mathrm{p} 450$. Immune related signalling pathways enriched in

328 KEGG analysis included IL-17 signaling, RIG-I-like receptor signaling, TNF signaling and

329 NOD-like receptor signaling (Fig. 7, File S3).

331 The heatmap revealed changes in gene expression between infected and non-infected sponges

332 (Fig. 6). We found that multiple loci of DBH-like and cytochrome P450-like monooxygenases,

333 glutathione S-transferases, copper transporting ATPases, and alcohol dehydrogenases were

334 among those upregulated in sponges infected with algal symbionts. Other noteworthy loci with

335 increased expression in symbiotic infected sponges include leukotrienes, cholesterol 24-

336 hydroxylase, L-amino-acid oxidase, sodium/potassium ATPase, and nmrA-like family domain-

337 containing protein 1. Genes involved in lysosomes/phagosomes, endocytosis, or autophagy (e.g.,

338 tartrate-resistant acid phosphatase type 5-like, cathepsin L, deleted in malignant brain tumors 1)

339 were among those increased in expression during uptake of symbionts. Genes involved in sugar

340 metabolism (e.g., protein phosphatase 1 regulatory subunit 3B-B-like, chitin synthase 3 ) and

341 signal transduction/gene regulation (e.g., transcriptional regulator Myc-A-like, cycloartenol-C- 
342 24-methyltransferase 1-like) were also represented among the genes with increased expression in

343 the symbiotic state.

345 While the majority of genes with decreased expression in symbiotic sponges are present at one

346 locus, ATP synthases, mucolipins, and E3 ubiquitin protein ligases occupy multiple loci. These

347 genes are known to be involved in ion transport and ubiquitination as well as other processes.

348 Genes involved in signal transduction or gene regulation (e.g., OAS1A, Ras-specific guanine

349 nucleotide-releasing factor 1, kielin/chordin-like protein, serine/threonine/tyrosine-interacting-

350 like protein 1, serine/threonine-protein kinase NIM1, NFX1-type zinc finger-containing protein

351 1) were often among those with lower expression in symbiotic sponges. We also found genes

352 involved in lysosomes/phagosomes (e.g., V-type proton ATPase, ceroid-lipofuscinosis neuronal

353 protein $6, \mathrm{~N}$-acylethanolamine acid amidase) among the genes that are downregulated during

354 symbiosis.

356 A few gene types had members that were either increased or decreased in response to infection

357 by native symbionts. Four distinct glutathione $\mathrm{S}$ transferase genes on three different

358 chromosomes $(1,9$, and 12) showed two to three fold level increases in expression in symbiotic

359 sponges, whereas expression in another glutathione S transferase (located on chromosome 5) was

360 decreased by 1.5 fold in symbiotic sponges. A complete lack of expression in symbiotic tissue

361 was observed for an elongation factor 1-gamma-like gene containing a glutathione S transferase

362 domain. Sponges infected with symbionts also had increased expression for two loci of the TNF

363 receptor-associated factor 3-like gene (both loci are clustered closely on chromosome 8). Other

364 genes that may be involved in NF-kB signalling were also upregulated including sequestosome- 
366 domain, and predicted cell death-inducing p53-target protein 1 that plays roles in regulating

367 TNF-alpha-mediated apoptosis. Expression of a TNF receptor-associated factor 4-like gene

368 (located on chromosome 1) and an uncharacterized protein predicted to be involved in TNF

369 signaling and apoptosis were decreased in symbiotic sponges.

371 Discussion

372 Freshwater sponge:algal symbiosis as tractable model

373 As articulated by Bosch, Guillemin \& McFall-Ngai (2019), the use of several laboratory

374 symbiosis model systems (“evolutionary 'experiments' in symbiosis") will help construct a more

375 complete picture of viable pathways towards stable intracellular residency and thus animal

376 evolution. We believe that E. muelleri is an excellent candidate to be a model system for these

377 types of studies. Its ubiquity around the globe and ease of collection make it widely available.

378 The freshwater sponge is easy to grow in the lab and harbors algal symbionts that can be

379 propagated in culture. Added benefits include the fact that this sponge has a recently sequenced

380 chromosomal level genome and other genomic and transcriptomic resources available (Kenny et

381 al., 2020). The fact that the mutualism is facultative, with the ability to culture the organisms

382 separately and conduct reinfection experiments (Figs. 1 \& 2), offers opportunities to study

383 pathways that permit long-term, stable residency within host cells. We have shown here that the

384 symbiotic algae can be tracked in sponge tissues via confocal (Figs. 3 \& 4) and electron

385 microscopy (Fig. 4). 
387 While marine sponges are important models of animal-microbe symbioses, both because they

388 produce pharmaceutically important bioactive compounds and due to their potential to illuminate

389 conserved mechanisms of host-microbe interactions in the basal metazoa (reviewed in Pita,

390 Fraune \& Hentschel, 2016), freshwater sponges should be considered as models to understand

391 possible convergent pathways leading to intra- and extracellular symbioses. Freshwater sponges

392 also have the added benefit of having many adaptations to freshwater systems (e.g., extreme

393 thermal tolerance, resilience in anoxic conditions, resistance to many pollutants, ability to

394 withstand desiccation, osmotic regulation). Thus, freshwater sponges have the potential to shed

395 light on symbiosis as well as a variety of important physiological features. Recent work by

396 Kenny et al., (2019) has already shown that freshwater sponges have extensive gene duplications

397 driving evolutionary novelty and have benefited from symbioses that allow them to live in

398 challenging conditions. Given that E. muelleri has a higher gene content than most animals,

399 nearly twice that of humans (Kenny et al., 2020), it may not be surprising to find a large number

400 of taxonomic specific genes among those that are differentially expressed. However, it has been

401 noted by others that taxonomically restricted genes (TRGs) could be key to the development of

402 species specific adaptive processes like endosymbiosis (Khalturin et al., 2009; Hamada et al.,

403 2018) and thus, these genes may be important in initiating or maintaining the symbioses in these

404 sponges. Our work to adapt E. muelleri as a model to forward these goals should impact our

405 future understanding of these important animals as well as the evolutionary mechanisms that

406 shape endosymbiosis. We focus in the following sections on some of the key findings.

407 
409 It is well documented that oxidative environments play key roles in regulating symbiotic

410 associations, and the interplay between regulators of redox biology have likely shaped the

411 evolution of symbioses across life forms (Moné, Monnin \& Kremer, 2014). Molecules involved

412 in redox homeostasis can mediate molecular communication between hosts and symbionts as

413 well as play roles in responses to toxic states with important pleiotropic roles for reactive oxygen

414 and nitrogen species during the establishment of symbioses. These roles include modulation of

415 cell division and differentiation, cellular signaling (e.g., NF-kappa B), kinase and phosphatase

416 activities, ion homeostasis $\left(\mathrm{Ca}_{2+}, \mathrm{Fe}_{2+}\right)$, and apoptosis/autophagy (Moné, Monnin \& Kremer,

417 2014). Recent work in Hydra-Chlorella models demonstrate that symbiosis-regulated genes

418 often include those involved in oxidative stress response (Ishikawa et al., 2016; Hamada et al.,

419 2018). Comparisons of gene expression in Paramecium bursaria with and without Chlorella

420 variabilis show significant enrichment of gene ontology terms for oxidation-reduction processes

421 and oxidoreductase activity as the top GO categories (Kodama et al., 2014).

423 Given that endosymbionts are known to create reactive oxygen species (ROS) that can lead to

424 cellular, protein, and nucleic acid damage (Marchi et al., 2012) and that other symbiotic models

425 have highlighted the importance for the host in dealing with reactive oxygen and reactive

426 nitrogen species (RONS) (e.g., Richier et al., 2005; Lesser, 2006; Weis, 2008; Dunn et al., 2012;

427 Roth, 2014; Moné, Monnin \& Kremer, 2014; Hamada et al., 2018), it is not surprising that

428 oxidative reduction system genes are differentially regulated during symbiosis in these model

429 systems. For example, Ishikawa et al., (2016) show that while many genes involved in the

430 mitochondrial respiratory chain are downregulated in symbiotic Hydra viridissima, other genes

431 involved in oxidative stress (e.g., cadherin, caspase, polycystin) are upregulated. 
432 Metalloproteinases and peroxidases show both upregulation and downregulation in the Hydra

433 symbiosis, and Ishikawa et al. (2016) show that some of the same gene categories that are

434 upregulated in H. viridissima (i.e., peroxidase, polycystin, cadherin) exhibit more

435 downregulation in H. vulgaris, which is a more recently established endosymbiosis. Hamada et

436 al., (2018) also found complicated patterns of upregulation and downregulation in oxidative

437 stress related genes in Hydra symbioses. They found that contigs encoding metalloproteinases

438 were differentially expressed in symbiotic versus aposymbiotic $H$. viridissima.

440 We identified a strong indication for the role of oxidative-reduction systems when E. muelleri is

441 infected with Chlorella symbionts (Figs. 6 \& 7). While our RNASeq dataset comparing

442 aposymbiotic with symbiotic E. muelleri also show differentially expressed cadherins, caspases,

443 peroxidases, methionine-r-sulfoxide reductase/selenoprotein, and metalloproteinases, the

444 expression differences for this suite of genes was not typically statistically significant at the 24

445 hour post-infection time point (File S2). We find two contigs with zinc metalloproteinase-

446 disintegrin-like genes and one uncharacterized protein that contains a caspase domain (cysteine-

447 dependent aspartate-directed protease family) that are upregulated at a statistically significant

448 level as well as one mitochondrial-like peroxiredoxin that is down regulated. Thus, like in the

449 Hydra:Chlorella system, a caspase gene is upregulated and a peroxidase is downregulated.

450 However, some of the differentially regulated genes we found that are presumed to be involved

451 in oxidation reduction systems are different than those highlighted in the Hydra:Chlorella

452 symbiosis. Multiple contigs containing DBH-like monooxygenases and cytochrome p450 4F1-

453 like genes were increased in expression in symbiotic states in E. muelleri. Most of these genes

454 are known to be involved in cellular oxidation-reduction systems that maintain homeostasis or 
455 act in detoxification. Oxidative stress responses have been noted in other hosts with

456 photosynthesizing algal symbionts and may be used to deal with the reactive oxygen species

457 (ROS) produced during photosynthesis (e.g., Richier et al., 2005; Lesser, 2006; Hamada et al.,

458 2018). Interestingly, in Aiptasia colonized with an opportunistic Symbiodinium trenchii

459 compared to the same corals colonized by their native symbionts, S. minutum, upregulation of

460 two cytochrome P450 monooxygenases was found as well as a higher abundance of arachidonic

461 acid (Matthews et al., 2017). The authors speculate that this difference in lipid signaling is a

462 result of an oxidative stress response to the non-native symbiont, but the specific role for these

463 molecules in this system remains unclear. We do not see the wholesale upregulation of

464 monooxygenases, as we also find that a flavin-containing monooxygenase is downregulated in

465 the symbiotic state.

466

467 We find four loci containing distinct glutathione S transferase (GST) genes to be upregulated in

468 E. muelleri infected with green algal symbionts, and one loci containing a GST gene to be

469 downregulated during symbiosis. Interestingly, we have also noted upregulation of a GST in the

470 marine sponge C. varians infected with native Symbiodinium (manuscript in prep). Our

471 observation of upregulation of some GSTs and downregulation of other GSTs in sponges is

472 enigmatic given that others seem to have found these genes to be mostly downregulated during

473 symbiosis. Hamada et al., (2018) show that a GST gene is downregulated in the $H$.

474 viridissima:Chlorella symbiosis. A GST was also downregulated in the symbiotic sea anemone

475 A. viridis (Ganot et al., 2011) and in the coral $A$. digitifera infected with a competent

476 Symbiodinium strain (Mohamed et al., 2016). Kodama et al., (2014) showed that multiple GST

477 genes are downregulated in P. bursaria with Chlorella symbionts as compared to the symbiont 
478 free Paramecium. Based on observed cytological phenomena, Kodama et al., (2014) suggest

479 these proteins are involved in the maintenance of the symbiosis given that the presence of algal

480 symbionts minimizes photo-oxidative stress.

481

482 Regardless of the precise role for regulation of GSTs during endosymbiosis, the connection

483 between glutamine supply and synthesis in both the Paramecium (He et al., (2019) and Hydra

484 (Ishikawa et al., 2016; Hamada et al., 2018) systems may be an important connection. While

485 Hydra most likely turn on glutamine synthetase for Chlorella to import nitrogen (Hamada et al.,

486 2018), glutamine may also be used by the animal for synthesis and excretion of glutathione in

487 cell growth and viability promotion or for ameliorating potential oxidative stress (Amores-

488 Sánchez \& Medina, 1999). Furthermore, while GSTs are best known for their role as

489 detoxification enzymes, they are known to carry out a variety of other functions including

490 peroxidase and isomerase activities, inhibition of Jun N-terminal kinase, binding to a range of

491 ligands, and several novel classes of non-mammalian GSTs have functions that are not related to

492 oxidative stress. Given the extensive gene duplication in freshwater sponges that has been

493 described (see Kenny et al., 2019; Kenny et al., 2020) it seems possible that some of the

494 duplicated GST genes have retained functional overlap as evidenced by their co-regulation

495 during symbiosis, but others may have diverged to gain different functions. Investigating the role

496 of GSTs in symbiosis regulation and dysregulation is important for uncovering new facets of

497 host-symbiont interactions. 
500 Interpartner recognition is a key component of stable symbiotic partnerships, and host innate

501 immunity likely plays a role in determining which microbes are targeted for destruction and

502 which avoid detection (Weis, 2019). The E. muelleri genome possesses a variety of innate

503 immunity genes and the upregulation of these genes occurs at stage 5 of development when the

504 sponges have a fully organized body with ostia, canals, chambers and osculum giving them an

505 ability to interact with the outside environment (Kenney et al., 2020). Given that innate

506 immunity has been shown to play a role in coral-dinoflagellate symbiosis and the holobiont

507 (reviewed in Weis, 2019) as well as in Hydra:Chlorella symbiosis (Hamada et al., 2018), we

508 hypothesized that innate immune genes would be among those differentially regulated during the

509 early stages of symbiosis.

511 It is well known from cnidarian-algal symbioses that microbe-associated molecular pattern

512 (MAMP)-pattern recognition receptor (PRR) interactions are key signals playing roles in

513 symbiont recognition and possibly maintenance of the association (reviewed in Davy, Allemand

514 \& Weis, 2012). We found at least one gene involved in PRR signaling pathways (i.e., deleted in

515 malignant brain tumors 1 protein-like; dmbt1) to be expressed in symbiotic tissue, with no

516 expression in aposymbiotic sponges. Another dmbt1-like gene containing several scavenger

517 receptor cysteine-rich (SRCR) domains was decreased in expression in infected tissue. In

518 addition to dmbt1-like genes, we find several other genes that may have associated scavenger

519 receptor activity to be differentially expressed in apopsymbiotic compared to symbiotic $E$.

520 muelleri, including a tolloid-like protein (dorsal-ventral patterning tolloid-like protein 1) and

521 several sponge-specific uncharacterized proteins (Em0017g780a, Em0083g1a, Em0017g784a,

522 Em0742gla - all of which were downregulated). It is possible that these PRRs play an important 
523 role in freshwater sponge-green algal recognition. Dmbt1 is a multiple SRCR domain containing

524 glycoprotein implicated in immune defense and epithelial differentiation (Mollenhauer et al.,

525 2000). Scavenger receptors are a class of PRRs that may function in recognition and regulation

526 in cnidarian-Symbiodinium symbioses (Weis, 2019). We previously showed that dmbt1

527 exhibited increased expression in aposymbiotic Cliona varians compared to C. varians infected

528 with its Symbiodinium symbiont (Riesgo et al., 2014). Dmbt1 is downregulated upon bacterial

529 challenge in oysters (McDowell et al., 2014) and the coral Acropora millepora (Wright et al.,

530 2017). In the case of $A$. millepora, it was suggested that dmbtl may play a role in maintaining

531 symbiotic associations with commensal microbes. In addition to SRCR domains, this dmbtl gene

532 also contains a calcium-binding EGF-like domain characteristic of membrane-bound proteins

533 that require calcium binding for protein-protein interactions.

535 Other molecules may also play a role in pattern recognition. For example, we observed decreased 536 expression of two different sushi, von Willebrand factor type A genes. These types of

537 complement control domain containing proteins (CCP) are often involved as pattern recognition

538 molecules in determining "self" vs. "non-self." The multiple CCP we found have receptor-ligand

539 interaction regions, and their downregulation suggests potential influence of the symbiont on

540 host expression patterns. As regulators of complement activation, CCPs can protect cells by

541 interacting with components of the complement system or through activation of immune cells

542 and processing of immune complexes when dealing with microbes and other foreign materials

543 (Hourcade, Holers \& Atkinson, 1989). 
545 We also identified 15 differentially regulated contigs included in the KEGG enrichment data set

546 that were involved in the nucleotide-binding oligomerization domain-like receptor (NLR)

547 signaling pathway. These NLR are important components of innate immunity involved in

548 cytoplasmic recognition of pathogen- and damage-associated molecular patterns (PAMPs and

549 DAMPs, respectively) that specifically recognize "non-self" components of the cell (Creagh and

550 O’Neill 2006). The NLR signalling pathway initiates signaling cascades that lead to regulation of

551 NF-kB and MAPK pathways. One of the genes associated with NOD-like receptor signaling is

552 Oas1a, which was downregulated in our symbiotic sponges. Oas 1a is an interferon-induced,

553 dsRNA-activated antiviral enzyme that plays roles in innate immunity and apoptosis. In addition

554 to the typical 2'-5'-oligoadenylate synthetase 1 and Nucleotidyltransferase (NT) domains, the

555 Oas 1-like gene that we found contains a TPR repeat (signal transduction) domain as well as three

556 MYND finger domains, a probable pectinesterase domain, and two parallel beta helix regions

557 that share some similarity with pectate lyases. Whether pectin-moieties on the surface of the

558 symbiont are a target, and thus involved in symbiont acquisition, remains to be seen. Three

559 contigs related to MAPK signaling were also differentially regulated, including the Ras-specific

560 guanine nucleotide-releasing factor 1 which was decreased in expression in symbiotic $E$.

561 muelleri. Further experiments will be needed to ascertain how these pathways are involved in

562 initial uptake or maintenance of the symbiosis.

563

564 We found differentially expressed contigs related to innate immunity and apoptosis functions. In

565 particular, upregulation of two TNF receptor-associated factor 3-like genes and downregulation

566 of one TNF receptor-associated factor 4-like gene suggests a role for immune function or

567 apoptosis. TNF receptor-associated factor 4-like genes regulate activation of NF-kappa-B in 
response to signaling through Toll-like receptors whereas TNF receptor-associated factor 3-like genes tend to act as negative regulators of NF-kappa-B activity; both are involved in apoptotic

570 processes. We observed 1.) upregulation of a tartrate-resistant acid phosphatase type 5-like gene

571 in symbiotic tissue, which has GO categorization of negative regulation of tumor necrosis factor

572 (TNF) production; 2.) upregulation of cell death-inducing p53-target protein 1, which is known

573 to regulate TNF-alpha-mediated apoptosis; and 3.) upregulation of sequestosome-1, an

574 autophagosome cargo protein that is also known to regulate TNF receptor associated factors as

575 well as NF-kappa-B in some cellular contexts (Kim \& Ozato 2009). In addition to these genes,

576 we found other contigs with transcripts predicted to be involved in Toll-like receptor/NF-kappa-

$577 \mathrm{~B} / \mathrm{TNF}-$ receptor signaling and apoptosis amongst the sponge-specific uncharacterized and/or

578 predicted proteins that are differentially regulated in symbiotic states (File S2; Em0002g1214a,

579 Em0023g342a, Em0084g5a ). The coral-Symbiodinium literature provides evidence that

580 symbionts may be modulating the host immune response via repression of NF-kappa-B (e.g.,

581 Weis, 2019), and while more work will need to be done to determine if NF-kappa-B function is

582 repressed, our data suggests the involvement of the TNF pathway in modulating the symbiosis.

\section{Nitrogen metabolism}

585 Nitrogen has long been suspected to be a key factor in the regulation of symbiont populations in

586 hosts (Radecker et al. 2015), though regulatory connections between host and symbiont are

587 generally poorly understood. For photosynthetic symbionts, nitrogen demands are elevated due

588 to the photosynthetic apparatus, and nitrogen metabolism is a key feature of digestive processes

589 of heterotrophic hosts. Thus, there seem to be opportunities for host:symbiont coevolutionary

590 specialization in terms of nitrogen metabolic integration. 
592 In the Hydra:Chlorella symbiosis, glutamine synthetase (GS-1) expression was found to be

593 elevated in host tissue when Chlorella symbionts were present and when the host was exposed to

594 maltose (Hamada et al., 2018). Indeed, GS-1 was one of the four main genes shown to be

595 specifically upregulated in H. viridissima by the presence of Chlorella symbionts. Hamada et al.

596 (2018) demonstrated that the symbiotic Chlorella could not use nitrite and ammonium as

597 nitrogen sources, and instead relied upon Hydra for nitrogen assimilation through the action of

598 glutamine synthetase and the uptake and processing of ammonium to glutamine. While we do

599 not find glutamine synthetase to be upregulated in E. muelleri (at least not at $24 \mathrm{hr}$ post-

600 infection), we do find an asparagine synthetase (File S2) to be significantly increased in

601 expression in symbiotic compared to aposymbiotic sponges.

602

603 Two primary models have been proposed to explain hypothesized use and uptake of nitrogen in

604 symbioses involving heterotrophic hosts and phototrophic symbionts (see Wang and Douglas

605 1998). The first is the straightforward hypothesis that symbionts assimilate nitrogenous waste

606 (primarily ammonium) from the host and translocate it back to the host in other forms. The

607 second is the more complicated hypothesis that symbiont-derived carbon compounds reduce host

608 catabolism of nitrogenous compounds. Our data do not permit favoring one of these hypotheses,

609 but the potential regulation of a key enzyme in nitrogenous pathways deserves greater attention

610 given the importance of this element to photosynthetic efficiency and as a vehicle for

611 host:symbiont integration.

612 


\section{Conclusions}

615 We demonstrate the utility of a Ephydatia muelleri:chlorophyte symbiosis to identify features of

616 host cellular and genetic responses to the presence of intracellular algal partners. Freshwater

617 sponges and their symbiotic partners are easy to maintain under laboratory conditions, and the

618 genomic and transcriptomic data available for the host offer powerful experimental

619 opportunities. The freshwater sponge system also offers an important comparative perspective

620 when placed in the context of work done with Paramecium, Hydra, and other

621 heterotrophic:phototrophic symbioses. Our work demonstrates that freshwater sponges offer

622 many tractable qualities to study features of intracellular occupancy and thus meet many criteria

623 desired for a model system.

\section{Acknowledgements}

626 This work was supported by funding from the National Science Foundation (Award \#1555440)

627 to ALH and MSH and by an Institutional Development Award (IDeA) from the National

628 Institute of General Medical Sciences of the National Institutes of Health under grant number

629 P20GM103423. We are thankful to Omar Quintero for assistance with confocal microscopy.

631 References

632 Amann R, Springer N, Schönhuber W, Ludwig W, Schmid EN, Müller KD, Michel R.

633 1997. Obligate intracellular bacterial parasites of acanthamoebae related to Chlamydia

634 spp. Appl Environ Microbiol 63, 115-121 
636 Amores-Sánchez MI, Medina MÁ. 1999. Glutamine, as a Precursor of Glutathione, and

637 Oxidative Stress. Molecular Genetics and Metabolism 67: 100-105. DOI:

$638 \quad 10.1006 /$ mgme.1999.2857.

639

640 Avila-Ospina L, Marmagne A, Talbotec J, Krupinska K, Masclaux-Daubresse C. 2015.

641 The identification of new cytosolic glutamine synthetase and asparagine synthetase

642 genes in barley (Hordeum vulgare L.), and their expression during leaf senescence."

643 Journal of Experimental Botany 66: 2013-26. DOI: 10.1093/jxb/erv003.

644

645 Barbeau MA, Reiswig HM, Rath LC. 1989. Hatching of freshwater sponge gemmules

646 after low temperature exposure: Ephydatia mülleri (Porifera: Spongillidae). Journal of

647 Thermal Biology 14:225-231. DOI: https://doi.org/10.1016/0306-4565(89)90009-0.

649 Bordenstein SR, Theis KR. 2015. Host Biology in Light of the Microbiome: Ten

650 Principles of Holobionts and Hologenomes. PLoS Biology 13:e1002226. DOI:

651 https://doi.org/10.1371/journal.pbio.1002226.

653 Bosch TCG, Guillemin K, McFall-Ngai M. 2019. Evolutionary “Experiments” in

654 Symbiosis: The Study of Model Animals Provides Insights into the Mechanisms

655 Underlying the Diversity of Host-Microbe Interactions. Bioessays 1800256. DOI:

$65610.1002 /$ bies.201800256. 
658 Brøndsted A, Brøndsted HV. 1953. The effect of symbiontic zoochlorellae on the

659 germination rate of gemmules of Spongilla lacustris (L.). Dansk Naturhistorisk Forening

660 Videnskabelige Meddelelser 115:133-144.

661

662 Brøndsted HV, Løvtrup E. 1953. The respiration of sponge gemmules without and with

663 symbiotic unicellular algae. Dansk Naturhistorisk Forening Videnskabelige Meddelelser

$664 \quad 115: 145-157$.

665

666 Capdevila JH, Falck JR, Harris RC. 2000. Cytochrome P450 and arachidonic acid

667 bioactivation: Molecular and functional properties of the arachidonate monooxygenase.

668 Journal of Lipid Research 41:163-181.

669

670 Clark TJ, Friel CA, Grman E, Shachar-Hill Y, Friesen M. 2017. Modelling nutritional

671 mutualisms: challenges and opportunities for data integration. Ecology Letters 20:1203-

672 1215. DOI: https://doi.org/10.1111/ele.12810.

673

674 Creagh EM, O'Neill LA. 2006. TLRs, NLRs and RLRs: a trinity of pathogen sensors that

675 cooperate in innate immunity. Trends in Immunology 27: 352-357.

676

677 Davy SK, Allemand D, Weis VM. 2012. Cell biology of cnidarian-dinoflagellate

678 symbiosis. Microbiology and Molecular Biology Reviews 76:229-261. DOI:

679 10.1128/MMBR.05014-11. 
681 Douglas AE. 2010. The Symbiotic Habit. Princeton: Princeton University Press.

682

683 Dunn SR, Pernice M, Green K, Hoegh-Guldberg O, Dove SG. 2012. Thermal stress

684 promotes host mitochondrial degradation in symbiotic cnidarians: are the batteries of

685 the reef going to run out? PLoS One 7:e39024. DOI:

686 https://doi.org/10.1371/journal.pone.0039024.

688 Filgueiras LR, Serezani CH, Jancar, S. 2015. Leukotriene B4 as a potential therapeutic 689 target for the treatment of metabolic disorders. Frontiers in Immunology 6: 515. DOI:

690 https://doi.org/10.3389/fimmu.2015.00515

691

692 Frost TM, Williamson CE. 1980. In Situ Determination of the Effect of Symbiotic Algae

693 on the Growth of the Freshwater Sponge Spongilla Lacustris. Ecology 61:1361-1370.

694

695 Ganot P, Moya A, Magnone V, Allemand D, Furla P, Sabourault C. 2011. Adaptations to 696 endosymbiosis in a cnidarian-dinoflagellate association: differential gene expression 697 and specific gene duplications. PLoS Genetics 7: e1002187. DOI:

698 10.1371/journal.pgen.1002187.

700 Gilbert JJ, Allen HL. 1973a. Studies on the physiology of the green freshwater sponge

701 Spongilla lacustris: primary productivity, organic matter, and chlorophyll content.

702 Verhandlungen Internationale Vereinigung fur Theoretische und Angewandte 
705 Gilbert JJ, Allen HL. 1973b. Chlorophyll and primary productivity of some green,

706 freshwater sponges. Internationale Revue der Gesamten Hydrobiologie 58:633- 658.

708 Hall C, Rodriguez M, Garcia J, Posfai D, DuMez R, Wictor E, Quintero O, Hill MS,

709 Rivera AS, Hill A L. 2019. Secreted frizzled related protein is a target of PaxB and plays

710 a role 956 in aquiferous system development in the freshwater sponge, Ephydatia

711 muelleri. PLOS ONE 14, 957 e0212005. DOI:

712 https://doi.org/10.1371/journal.pone.0212005.

713

714 Hamada M, Schröder K, Bathia J, Kürn U, Fraune S, Khalturina M, Khalturin K,

715 Shinzato C, Satoh N, Bosch TCG. 2018. Metabolic co-dependence drives the

716 evolutionarily ancient Hydra-Chlorella symbiosis. eLife. 7:e35122. DOI:

717 10.7554/eLife.35122.

718

719 He M, Wang J, Fan X, Liu X, Shi W, Huang N, Zhao F, Miao M. 2019. Genetic basis for

720 the establishment of endosymbiosis in Paramecium. The ISME Journal 13: 1360-1369.

721 DOI: https://doi.org/10.1038/s41396-018-0341-4.

722

723 Hentschel U, Steinert M, Hacker J. 2000. Common molecular mechanisms of symbiosis

724 and pathogenesis. Trends in Microbiology 8, 226 - 231. DOI:10.1016/S0966-

$725842 \times(00) 01758-3$. 
727 Hill MS. 2014. Production possibility frontiers in phototroph:heterotroph symbioses:

728 trade-offs in allocating fixed carbon pools and the challenges these alternatives present

729 for understanding the acquisition of intracellular habitats. Frontiers in Microbiology 5 ,

730 357. DOI: 10.3389/fmicb.2014.00357.

732 Hill MS, Hill AL 2012. The magnesium inhibition and arrested phagosome hypotheses:

733 new perspectives on the evolution and ecology of Symbiodinium symbioses. Biological

734 Reviews 87, 804-821. DOI: https://doi.org/10.1111/j.1469-185X.2012.00223.x.

735

736 Hoshina R, Imamura N. 2008. Multiple Origins of the Symbioses in Paramecium

737 bursaria. Protist 159: 53-63.

738

739 Hourcade D, Holers VM, Atkinson JP. 1989. The Regulators of Complement Activation

740 (RCA) Gene Cluster. In: Dixon FJ, ed. Advances in Immunology, Academic Press, 45:

741 381-416. DOI: https://doi.org/10.1016/S0065-2776(08)60697-5.

743 Ishikawa M, Yuyama I, Shimizu H, Nozawa M, Ikeo K, Gojobori T. 2016. Different

744 Endosymbiotic Interactions in Two Hydra Species Reflect the Evolutionary History of

745 Endosymbiosis. Genome Biology and Evolution 8: 2155-2163. DOI:

$746 \quad 10.1093 / g b e / e v w 142$.

748 Karakashian SJ. 1963. Growth of Paramecium bursaria as influenced by the presence 749 of algal symbionts. Physiological Zoology 36:52-68. 
751 Kenny NJ, Plese B, Riesgo A, Itskovich VB. 2019. Symbiosis, Selection, and Novelty:

752 Freshwater Adaptation in the Unique Sponges of Lake Baikal. Molecular Biology and

753 Evolution 36:2462-2480. DOI: 10.1093/molbev/msz151.

755 Kenny NJ, Francis WR, Rivera-Vicéns RE, Jurval K, de Mendoza A, Díez-Vives C,

756 Lister R, Bezares-Calderon L, Grombacher L, Roller M, Barlow LD, Camilli S, Ryan JF,

757 Wöheide G, Hill AL, Riesgo A, Leys SP. 2020. Tracing animal genomic evolution with

758 the chromosomal-level assembly of the freshwater sponge Ephydatia muelleri. Nature

759 Communications 11:3676. DOI: https://doi.org/10.1038/s41467-020-17397-w.

761 Khalturin K, Hemmrich G, Fraune S, Augustin R, Bosch TC. 2009. More than just

762 orphans: are taxonomically restricted genes important in evolution? Trends in Genetics

763 25:404-413. DOI: 10.1016/j.tig.2009.07.006.

765 Kim JY, Ozato K. 2009. The sequestosome 1/p62 attenuates cytokine gene expression

766 in activated macrophages by inhibiting IFN regulatory factor 8 and TNF receptor-

767 associated factor 6/NF-kappaB activity. Journal of Immunology 182:2131-2140.

768 DOI:10.4049/jimmunol.0802755.

770 Kim D, Langmead B, Salzberg SL. 2015. HISAT: a fast spliced aligner with low memory

771 requirements. Nature Methods 12:357-360. DOI: 10.1038/nmeth.3317. 
773 Kodama Y, Fujishima M. 2010. Secondary symbiosis between Paramecium and

774 Chlorella cells. In: Jeon KW, ed. International Review of Cell and Molecular Biology Vol.

775 279, San Diego, Burlington, London, Amsterdam: Elsevier, pp. 33-77.

776

777 Kodama Y, Suzuki H, Dohra H, Sugii M, Kitazume T, Yamaguchi K, Shigenobu S,

778 Fujishima M. 2014. Comparison of gene expression of Paramecium bursaria with and

779 without Chlorella variabilis symbionts. BMC Genomics 15:183. DOI:

780 https://doi.org/10.1186/1471-2164-15-183.

781

782 Kovacevic G. 2012. Value of the Hydra model system for studying symbiosis.

783 International Journal of Developmental Biology 56: 627-635. DOI:

$784 \quad 10.1387 / \mathrm{ijdb} .123510 \mathrm{gk}$.

785

786 Langmead B, Salzberg SL. 2012. Fast gapped-read alignment with Bowtie 2. Nature

787 Methods 9:357-359. DOI: https://doi.org/10.1038/nmeth.1923.

788

789 Lesser MP. 2006. Oxidative stress in marine environments: biochemistry and

790 physiological ecology. Annual Review of Physiology 68:253-278. DOI:

791 10.1146/annurev.physiol.68.040104.110001.

792

793 Leys S, Grombacher L, Hill A. 2019. Hatching and freezing gemmules from the

794 freshwater sponge Ephydatia muelleri. Protocols.io. DOI:

795 dx.doi.org/10.17504/protocols.io.863hzgn. 
797 Manconi R, Pronzato R. 2016. How to survive and persist in temporary freshwater?

798 Adaptive traits of sponges (Porifera: Spongillida): A review. Hydrobiologia 782:11-22.

799 DOI: https://doi.org/10.1007/s10750-016-2714-x.

801 Marchi S, Giorgi C, Suski JM, Agnoletto C, Bononi A, Bonora M, De Marchi E, Missiroli

802 S, Patergnani S, Poletti F, Rimessi A, Duszynski J, Wieckowski MR, Pinton P. 2012.

803 Mitochondria-ros crosstalk in the control of cell death and aging. Journal of Signal

804 Transduction 2012: 329635. DOI: https://doi.org/10.1155/2012/329635.

806 Margulis L. 1993. Origins of species: acquired genomes and individuality. Biosystems 807 31:121-125. DOI: https://doi.org/10.1016/0303-2647(93)90039-F.

Marin B, Klingberg M, Melkonian M. 1998. Phylogenetic Relationships among the

810 Cryptophyta: Analyses of Nuclear-Encoded SSU rRNA Sequences Support the

811 Monophyly of Extant Plastid-Containing Lineages. Protist 149: 265-276. DOI:

$812 \quad 10.1016 / S 1434-4610(98) 70033-1$.

814 Marin B, Palm A, Klingberg M, Melkonian M. 2003. Phylogeny and Taxonomic Revision 815 of Plastid-Containing Euglenophytes based on SSU rDNA Sequence Comparisons and 816 Synapomorphic Signatures in the SSU rRNA Secondary Structure. Protist 154: 99-145.

817 DOI: $10.1078 / 143446103764928521$. 
819 Martin M. 2011. Cutadapt removes adapter sequences from high-throughput

820 sequencing reads. EMBnet.journal 17:10-12. DOI: https://doi.org/10.14806/ej.17.1.200.

822 Matthews JL, Crowder CM, Oakley CA, Lutz A, Roessner U, Meyer E, Grossman AR, 823 Weis VM, Davy SK. (2017) Optimal nutrient exchange and immune responses operate

824 in partner specificity in the cnidarian-dinoflagellate symbiosis. Proceedings of the

825 National Academy of Sciences 114:13194-13199. DOI: 10.1073/pnas.1710733114.

827 McAuley DG, Longcore JR. 1988. Foods of juvenile Ring-Necked Ducks: Relationship

828 to wetland $\mathrm{pH}$. Journal of Wildlife Management 52: 177-185.

830 McDowell IC, Nikapitiya C, Aguiar D, Lane CE, Istrail S, Gomez-Chiarri M. 2014.

831 Transcriptome of American oysters, Crassostrea virginica, in response to bacterial

832 challenge: insights into potential mechanisms of disease resistance. PLoS One

833 98:e105097. DOI::10.1371/journal.pone.0105097.

835 Mohamed AR, Cumbo V, Harii S, Shinzato C, Chan CX, Ragan MA, Bourne DG, Willis

836 BL, Ball EE, Satoh N, Miller DJ. 2016. The transcriptomic response of the coral

837 Acropora digitifera to a competent Symbiodinium strain: the symbiosome as an arrested

838 early phagosome. Molecular Ecology 25:3127-3141. DOI:

839 https://doi.org/10.1111/mec.13659. 
841 Mollenhauer J, Herbertz S, Holmskov U, Tolnay M, Krebs I, Merlo A, Schrøder HD,

842 Maier D, Breitling F, Wiemann S, Gröne H-J, Poustka A. 2000. DMBT1 Encodes a

843 Protein Involved in the Immune Defense and in Epithelial Differentiation and Is Highly

844 Unstable in Cancer. Cancer Research 60:1704-1710.

846 Moné Y, Monnin D, Kremer N. 2014. The oxidative environment: a mediator of

847 interspecies communication that drives symbiosis evolution. Proceedings of the Royal

848 Society B 281:20133112. DOI: http://dx.doi.org/10.1098/rspb.2013.3112.

850 Muscatine L, Karakashian SJ, Karakashian MW. 1967. Soluble extracellular products of

851 algae symbiotic with a ciliate, a sponge and a mutant hydra. Comparative Biochemistry

852 and Physiology 20:1-12. DOI: https://doi.org/10.1016/0010-406X(67)90720-7.

854 Muscatine L, Hand C. 1958. Direct evidence for the transfer of materials from symbiotic

855 algae to the tissues of a coelenterate. Proceedings of the National Academy of

856 Sciences USA 44:1259-1263. DOI: 10.1073/pnas.44.12.1259.

858 Pertea M, Pertea GM, Antonescu CM, Chang TC, Mendell JT, Salzberg SL. 2015.

859 String Tie enables improved reconstruction of a transcriptome from RNA-seq reads.

860 Nature Biotechnology 33:290-295. DOI: https://doi.org/10.1038/nbt.3122. 
862 Pita L, Fraune S, Hentschel U. 2016. Emerging Sponge Models of Animal-Microbe

863 Symbioses. Frontiers in Microbiology 7:2102. DOI:

864 https://doi.org/10.3389/fmicb.2016.02102.

866 Peyer SM, Kremer N, McFall-Ngai MJ. 2018. Involvement of a host Cathepsin L in

867 symbiont-induced cell death. MicrobiologyOpen 7:e00632. DOI:10.1002/mbo3.632.

869 Radecker N, Pogoreutz C, Voolstra CR, Wiedenmann J, Wild C. 2015.

870 Nitrogen cycling in corals: The key to understanding holobiont functioning? Trends in

871 Microbiology, 23:490-497.

872

873 Rajević N, Kovacević G, Kalafatić M, Gould SB, Martin WF, Franjević D. 2015. Algal

874 endosymbionts in European Hydra strains reflect multiple origins of the zoochlorella

875 symbiosis. Molecular Phylogeny and Evolution 93:55-62. DOI:

$876 \quad$ 10.1016/j.ympev.2015.07.014.

878 Reiser W. ed. 1992. Algae and symbioses: plant, animals, fungi, viruses, interactions 879 explored. Biopress, Bristol.

881 Riesgo A, Peterson K, Richardson C, Heist T, Strehlow B, McCauley M, Cotman C, Hill

882 M, Hill A. 2014. Transcriptomic analysis of differential host gene expression upon

883 uptake of symbionts: as case study with Symbiodinium and the major bioeroding 
884 sponge Cliona varians. BMC Genomics 15: 376. https://doi.org/10.1186/1471-2164-15-

$885 \quad 376$.

886

887 Richier S, Furla P, Plantivaux A, Merle PL, Allemand D. 2005. Symbiosis-induced

888 adaptation to oxidative stress. Journal of Experimental Biology 208:277-285. DOI:

$88910.1242 /$ jeb.01368.

890

891 Rivera AS, Hammel JU, Haen KM, Danka ES, Cieniewicz B, Winters IP, Posfai D,

892 Wörheide G, Lavrov DV, Knight SW, Hill MS, Hill AL, Nickel M. 2011. RNA interference

893 in marine and freshwater sponges: actin knockdown in Tethya wilhelma and Ephydatia

894 muelleri by ingested dsRNA expressing bacteria. BMC Biotechnology 11:67. DOI:

$895 \quad 10.1186 / 1472-6750-11-67$.

896

897 Robinson MD, McCarthy DJ, Smyth GK. 2010. edgeR: a Bioconductor package for

898 differential expression analysis of digital gene expression data. Bioinformatics 26: 139-

899 140. DOI: https://doi.org/10.1093/bioinformatics/btp616.

900

901 Roth MS. 2014. The engine of the reef: photobiology of the coral-algal symbiosis.

902 Frontiers in Microbiology 422:1-22. DOI: 10.3389/fmicb.2014.00422.

903

904 Schenkelaars Q, Quintero O, Hall C, Fierro-Constain L, Renard E, Borchiellini C, Hill

905 AL. 2016. ROCK inhibition abolishes the establishment of the aquiferous system in 
906 Ephydatia muelleri (Porifera, Demospongiae). Developmental Biology 412:298-310.

907 DOI: 10.1016/j.ydbio.2016.02.026.

908

909 Schippers KJ, Nichols SA. 2018. Evidence of Signaling and Adhesion Roles for $\beta$ -

910 catenin in the sponge Ephydatia muelleri. Molecular Biology and Evolution 35: 1407-

911 1421. DOI: https://doi.org/10.1093/molbev/msy033.

912

913 Smith DC, Douglas AE. 1987. The biology of symbiosis. Edward Arnold, London. DOI:

914 https://doi.org/10.1017/S0025315400027491.

916 Stambler N. 2011. Zooxanthellae: the yellow symbionts inside animals. In: Dubinsky Z,

917 Stambler N, eds. Coral Reefs: An Ecosystem in Transition. Dordrecht: Springer 87-106.

918 DOI: https://doi.org/10.1007/978-94-007-0114-4_7.

920 Strekal TA, McDiffett WF. 1974. Factors affecting germination, growth, and distribution 921 of the freshwater sponge, Spongilla fragilis Leidy (Porifera). Biological Bulletin 146:267922 278. DOI:10.2307/1540623.

923

924 Strehlow B, McCauley M, Friday S, Hill M. 2016. The potential of azooxanthellate 925 poriferan hosts to assess the fundamental and realized Symbiodinium niche: Evaluating

926 a novel method to initiate Symbiodinium associations. Coral Reefs 35:1201-1212.

927 DOI:10.1007/s00338-016-1465-5. 
929 Wang J, Douglas AE. 1998. Nitrogen recycling or nitrogen conservation in an alga-

930 invertebrate symbiosis? Journal of Experimental Biology 201:2445-2453.

931

932 Weis VM. 2008. Cellular mechanisms of cnidarian bleaching: stress causes the collapse

933 of symbiosis. Journal of Experimental Biology 211:3059-3066. DOI:

934 10.1242/jeb.009597.

935

936 Weis VM. 2019. Cell Biology of Coral Symbiosis: Foundational Study Can Inform

937 Solutions to the Coral Reef Crisis. Integrative and Comparative Biology 594:845-855.

938 DOI:10.1093/icb/icz067.

939

940 Wilkinson CR. 1980. Nutrient translocation from green algal symbionts to the freshwater

941 sponge Ephydatia fluviatilis. Hydrobiologia 75:241-250. DOI:

942 https://doi.org/10.1007/BF00006488.

943

944 Williamson CE. 1977. Fluorescence identification of zoochlorellae: a rapid method for

945 investigating algae invertebrate symbioses. Journal of Experimental Zoology 202:187-

946 194. DOI: https://doi.org/10.1002/jez.1402020207.

947

948 Williamson, C. E. 1979. An ultrastructural investigation of algal symbiosis in white and

949 green Spongil/a lacustris (L.) (Porifera: Spongillidae). Transactions of the American

950 Microscopical Society 98:59-77. DOI: 10.2307/3225940. 
952 Windsor PJ, Leys SP. 2010. Wnt signaling and induction in the sponge aquiferous 953 system: evidence for an ancient origin of the organizer: polarity in sponges. Evolution

954 and Development 12:484-493. DOI: https://doi.org/10.1111/j.1525-142X.2010.00434.x.

955

956 Windsor Reid PJ, Matveev E, McClymount A, Posfai D, Hill AL, Leys SP. 2018. Wnt

957 signaling and polarity in freshwater sponges. BMC Evolutionary Biology 18:12. DOI:

958 https://doi.org/10.1186/s12862-018-1118-0.

959

960 Wright RM, Kenkel CD, Dunn CE, Shilling EN, Bay LK, Matz M. 2017. Intraspecific

961 differences in molecular stress responses and coral pathobiome contribute to mortality

962 under bacterial challenge in Acropora millepora. Scientific Reports 7:2609. DOI:

963 https://doi.org/10.1038/s41598-017-02685-1.

964

965 Wu H-L, Hseu R-S, Lin L-P. 2001. Identification of Chlorella spp. isolates using

966 ribosomal DNA sequences. Botanical Bulletin- Academia Sinica Taipei 42:115-121.

967

968

969

970

971

972

973

974 


\section{Figures}

976 Figure 1. Freshwater sponges in natural habitat growing at the outflow of the dam. Several

977 sponge species are present - some harbor green algae, some do not. (Inset) Example of sponge

978 harboring green algae. The sponge was growing on the underside of a rock, which has been

979 turned over. The portion of the sponge that would have been exposed to sunlight (bottom portion

980 of the sponge) is green due to the presence of Chlorella. Tissue protected from sunlight is devoid

981 of algae (top portion of sponge colony).

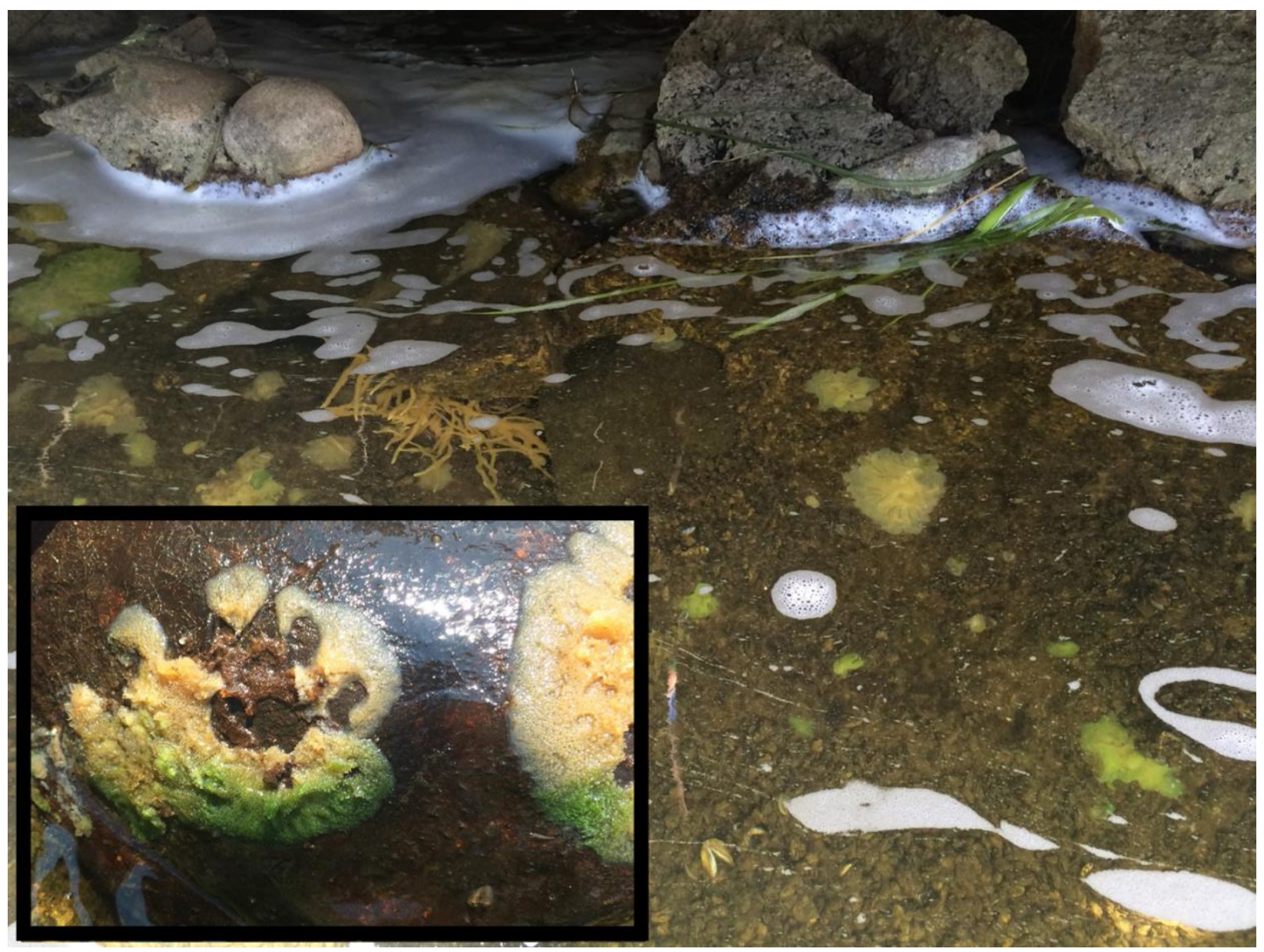


Figure 2. Infection of aposymbiotic E. muelleri sponges. A. Schematic of infection process. Inset

987 shows electron micrograph of Chlorella engulfment by sponge cell. B. E. muelleri without algae

988 (left) and 24 hours post-infection with Chlorella symbionts (right). O (osculum), C (canal).

A.)

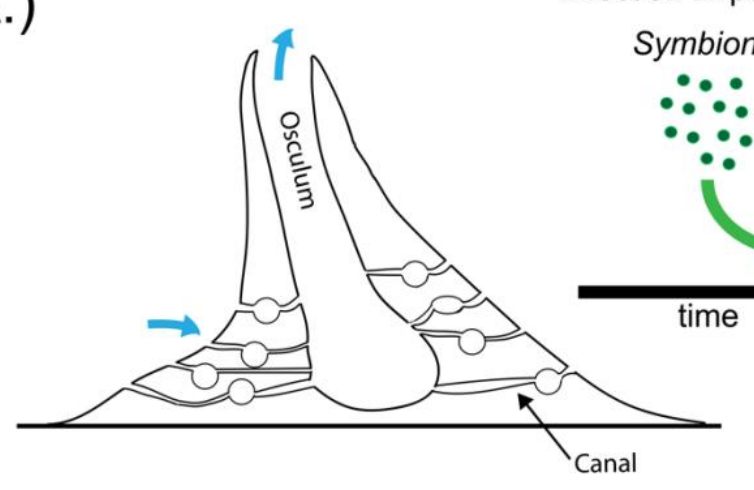

B.)

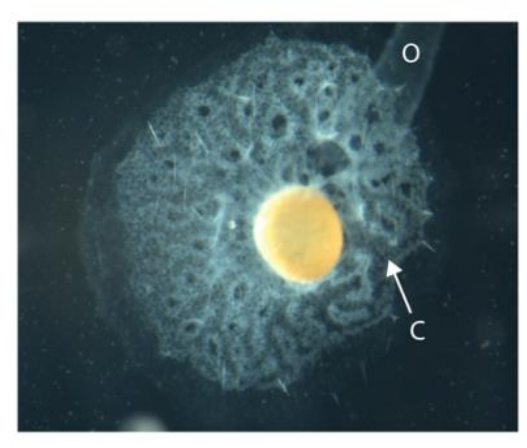

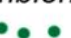

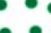

time
Infection Experiments Symbionts

$\because \bullet$

1

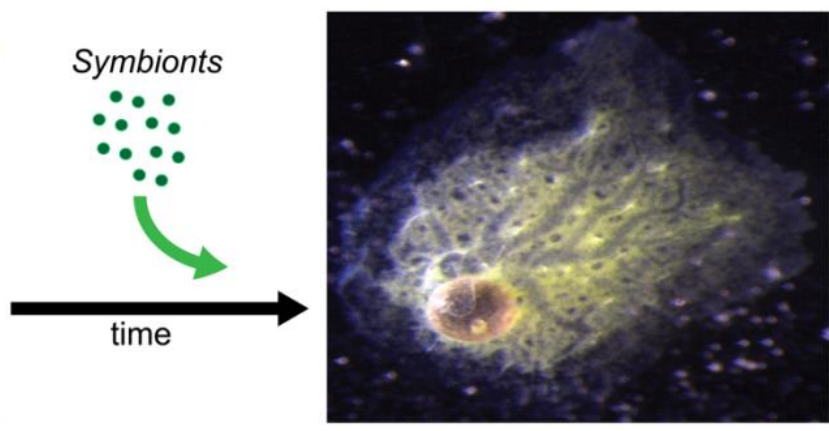


999 Figure 3. Confocal time series of E. muelleri choanoderm region after infection with Chlorella

1000 symbionts. A. Aposymbiotic E. muelleri. B. E. muelleri 4 hrs post-infection. C. E. muelleri 24

1001 hrs post-infection. D. E. muelleri 6 days post-infection. Note cells with multiple algae. Images

1002 show DNA in blue, F-actin in green, and autofluorescence of algal cells in red. Scale bars $30 \mu \mathrm{m}$.

1003
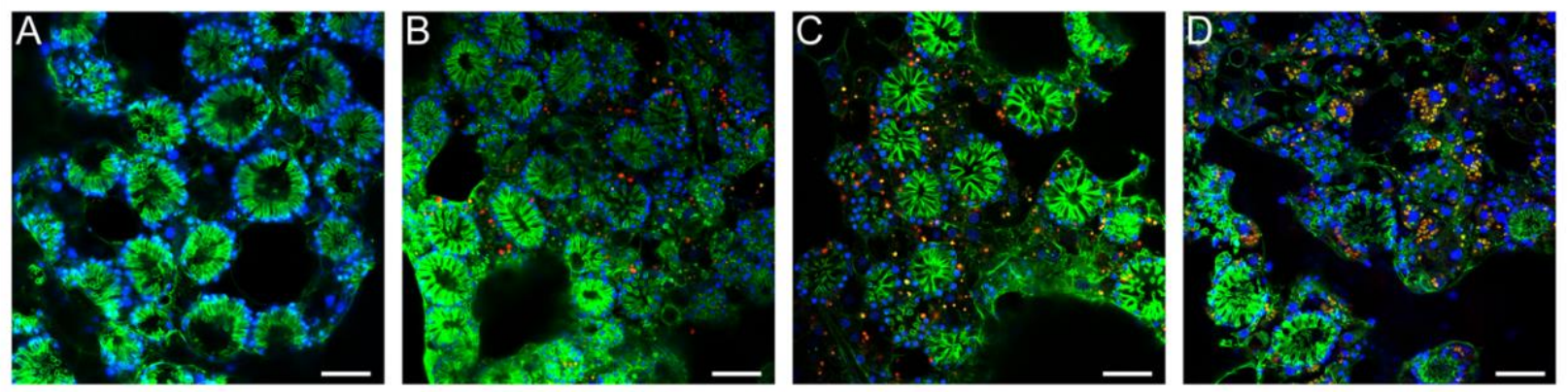

1004

1005

1006

1007

1008

1009

1010

1011

1012

1013

1014

1015

1016

1017 
1018 Figure 4. Transmission electron microscopy of intracellular Chlorella after E. muelleri

1019 infections. A. E. muelleri 4 hrs post-infection. B. Multiple infected cells 24 hrs post-infection. C.

1020 Once cell with multiple algal symbionts 24 hrs post-infection. Scale bars $2 \mu \mathrm{m}$.
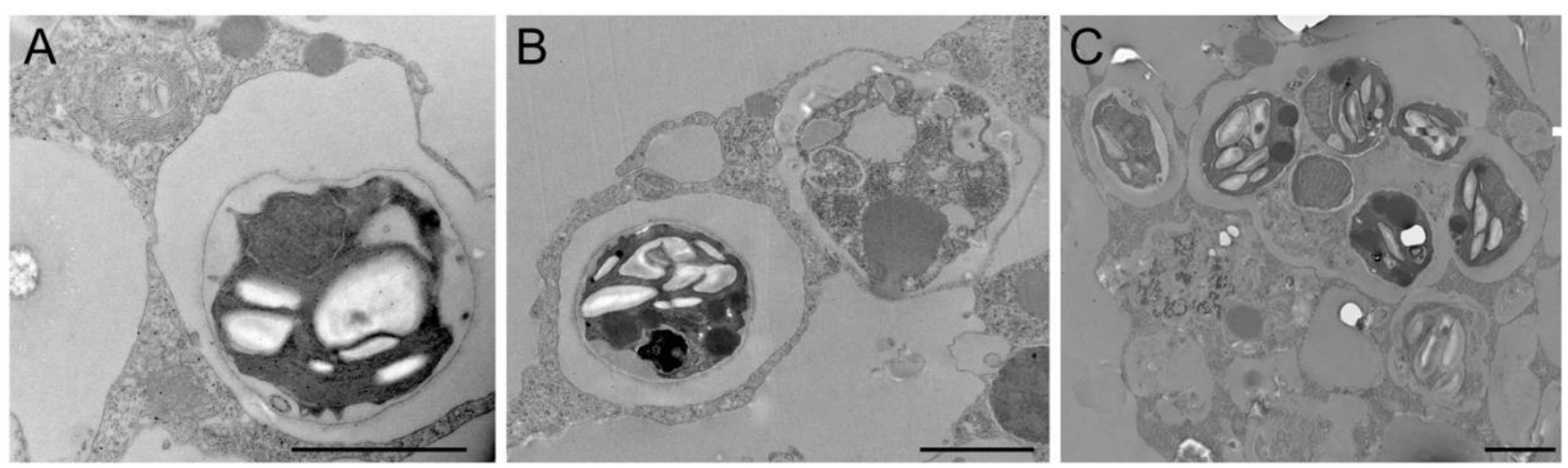

1022

1023

1024

1025

1026

1027

1028

1029

1030

1031

1032

1033

1034

1035

1036 
1037 Figure 5. Confocal image at 24 hrs post-infection showing multiple intracellular Chlorella

1038 symbionts in one sponge cell. Images show DNA in blue, F-actin in green, and autofluorescence

1039 of algal cells in red. Scale bars $20 \mu \mathrm{m}$.

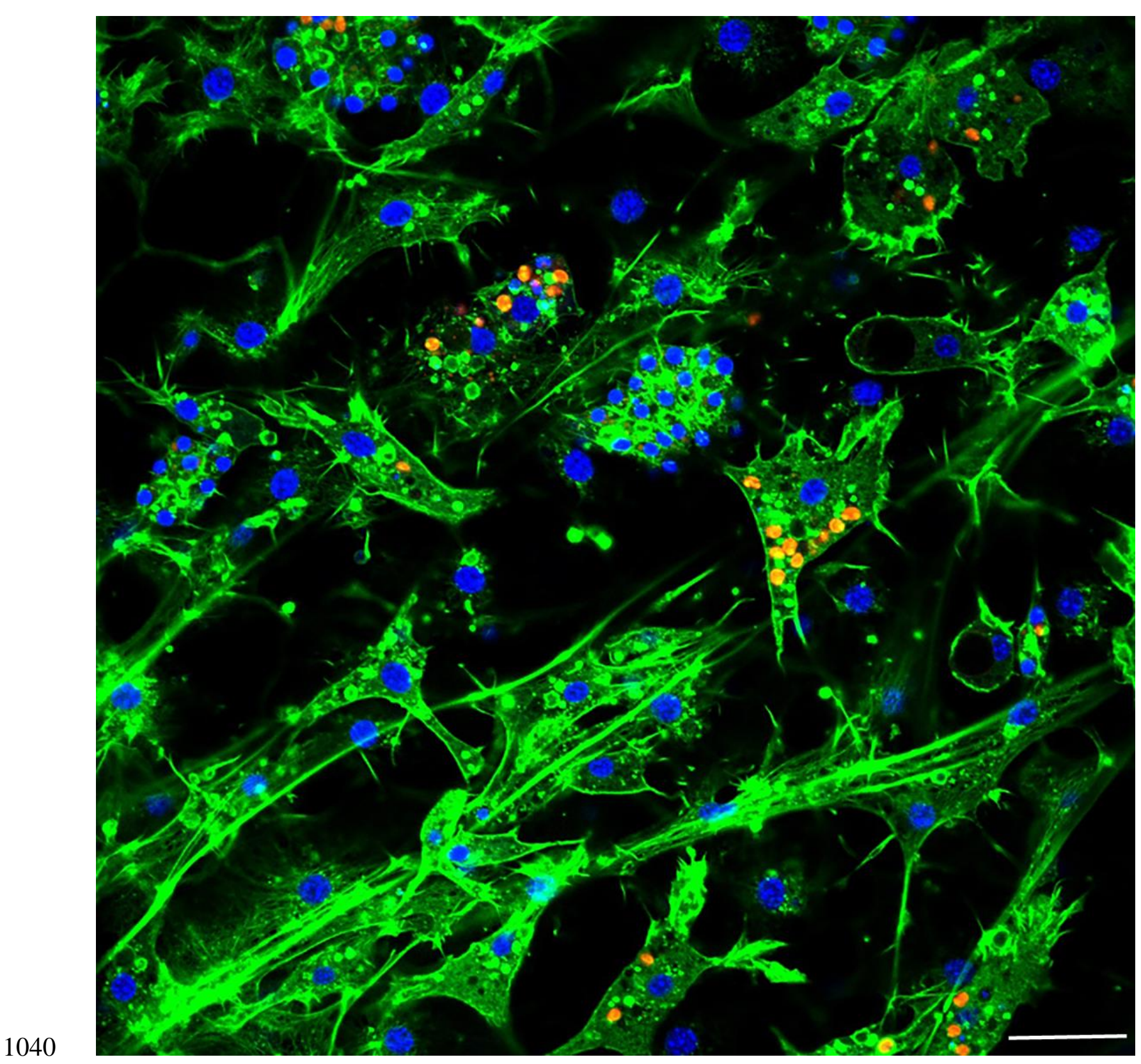


1044 Figure 6. Heatmap of differentially expressed genes in RNASeq analysis. Relative expression of

1045 differentially expressed genes (scale at right) are shown comparing triplicate samples for

1046 aposymbiotic and 24 hrs post-infected sponges. Gene IDs are provided at the right of each

1047 expression profile. 
bioRxiv preprint doi: https://doi.org/10.1101/2020.08.12.247908; this version posted August 13, 2020. The copyright holder for this preprint (which was not certified by peer review) is the author/funder. All rights reserved. No reuse allowed without permission.

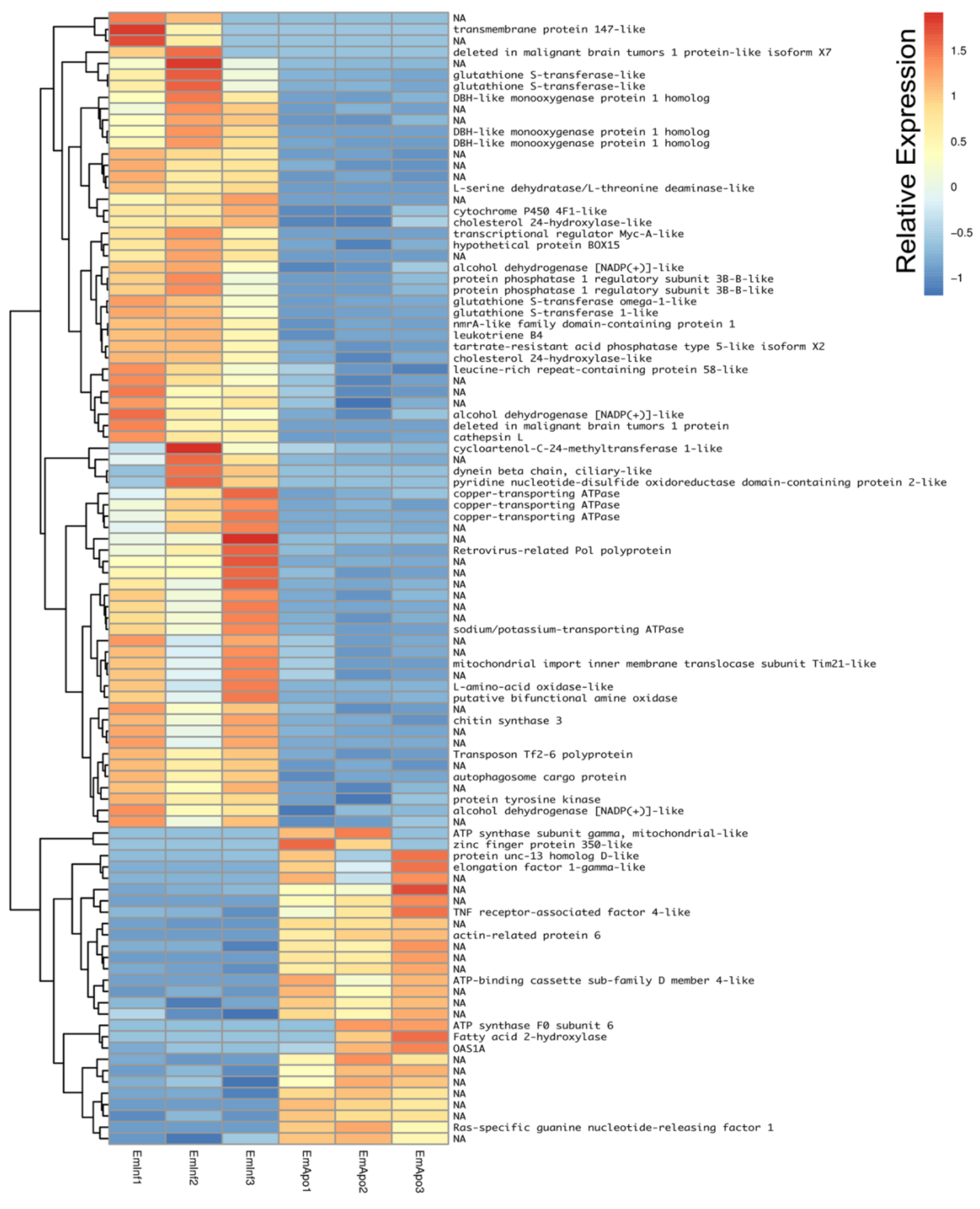


1051 Figure 7. Enrichment categories for Gene Ontology and KEGG. A. Gene ontology (GO)

1052 enrichment. B. KEGG enrichment across the differential gene expression analysis for

1053 aposymbiotic and $24 \mathrm{hrs}$ post-infection E muelleri.
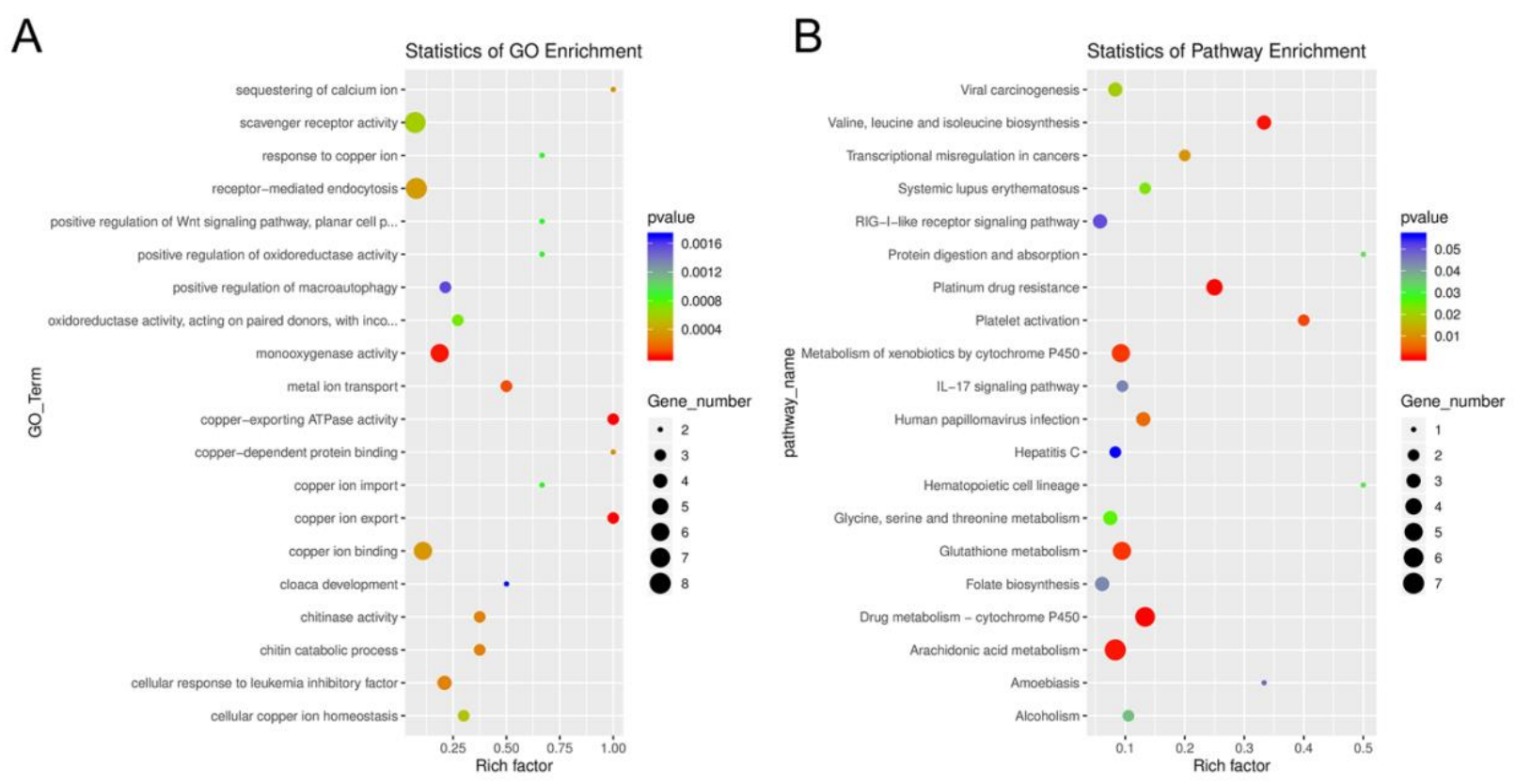